\title{
LOCALIZACIÓN Y ORIENTACIÓN DE EQUIPOS MÓVILES USANDO COLOR
}

\author{
Juan Sebastián Botero Valencia ${ }^{1}$ \\ Edilson Delgado Trejos ${ }^{2}$
}

\section{Resumen}

Uno de los mayores problemas en el desarrollo de interfaces móviles autónomas está ligado al desarrollo del sistema de localización. Es fundamental que se conozca la posición del móvil para cerrar el lazo de control sobre la estrategia que se use en la planeación de trayectorias. En este trabajo se presenta un algoritmo que individualiza dos conjuntos de Robots Móviles además de orientar y localizar cada móvil en el plano. Con este fin, se propone el uso de técnicas de segmentación por umbralización binaria en conjunto con operaciones lógicas. El sistema está compuesto por una cámara digital periférica que observa la totalidad del plano y un conjunto de Móviles que son controlados por un sistema maestro. Los móviles en su construcción física sólo cuentan con dos posibles direcciones de desplazamiento y rotación sobre su eje. A partir del estudio, se obtiene un algoritmo que identifica por equipos dos conjuntos de móviles y obtiene la posición y orientación individual. El sistema es complementado con una estrategia de predicción que detecta una posible colisión. Finalmente, se obtiene un sistema que identifica de forma cartesiana dos conjuntos de Robots Móviles y coordina su desplazamiento.

1 Ingeniero Electrónico. Estudiante de Maestría en Automatización y Control Industrial. Auxiliar de investigación. Instituto Tecnológico Metropolitano. Email: juanbotero@itm.edu.co

2 Ingeniero Electrónico. M. Sc. en Automatización Industrial. Ph. D. en Ingeniería LI Automática. Académico Investigador del Centro de Investigación, Instituto Tecnológico Metropolitano. Email: edilsondelgado@itm.edu.co 


\section{Palabras clave}

Color, umbralización, robot móvil, segmentación, trayectoria.

\section{Abstract}

One of the biggest problems in the development of autonomous mobile interfaces is linked to the development of tracking system. It is essential to know the position of the mobile to close the control loop on the strategy used in path planning. This paper presents an algorithm that identifies two sets of mobile robots as well as guiding and locating each mobile in the plane. To this end, we propose the use of segmentation by thresholding techniques in conjunction with binary logic operations. The system consists of a peripheral digital camera that observes the entire plane and a set of mobility, which are controlled by a master system. Mobile Robots in its physical construction are two possible directions of movement and rotation on its axis. From the study, was obtained an algorithm that identifies two sets of mobile equipment and obtains the position and orientation. The system is supplemented by a prediction strategy that detects a possible collision. Finally, we get a system that identifies two sets of Mobile Robots on a cartesian plane and manages their movement.

\section{Keywords}

Color, thresholding, mobile robot, segmentation, trajectory. 


\section{INTRODUCCIÓN}

La robótica móvil a despertado un interés relativamente reciente respecto al desarrollo de problemas de inteligencia colectiva, muchos problemas comunes se resuelven de manera más eficiente si se enfrentan de forma paralela como lo hacen los sistemas construidos con inteligencia de enjambre (Shoham \& Leyton-Brown, 2009). En general se puede decir que los elementos individuales de la inteligencia colectiva pueden ser más simples, lo que facilita su construcción. Inspirados en los sistemas biológicos, se observa como un gran conjunto de unidades simples organizadas resuelven un problema complejo a partir de el desarrollo de un sistema de reglas (Minati \& Pessa, 2007), también es posible que estos sistemas desarrollen las reglas que mejor se adapten a su entorno y que mejoren el sistema de control que inicialmente se planeó para ellos. Sin embargo, la dirección de elementos inteligentes que actúan en paralelo trae problemas de administración a los sistemas comunes de procesamiento serial.

Un móvil que se desplaza en un plano, implica varios problemas para el sistema de control; en primer lugar su ubicación, es decir, que basándose en algún tipo de sistema de coordenadas el sistema de control conozca su posición, aparte de esto, el sistema de control debe conocer su orientación, un indicador que ayude a correlacionar las órdenes de movimiento con el desplazamiento real. Por si esto fuera poco, el sistema de control también debe conocer el espacio que el robot necesita para maniobrar. Para planear trayectorias se deben tomar en cuenta variables adicionales (tiempo de respuesta del sistema), que como se muestra en (Arias \& León, 2007) puede implicar un tiempo tan alto, que haría imposible predecir una colisión con los móviles desplazándose a $0.3 \mathrm{~m} / \mathrm{s}$. Algunos sistemas de localización usan información relativa de la posición (Ortíz et al., 2008), el problema de estos es que obtienen la posición absoluta partiendo del conocimiento de la posición inicial y ciertas perturbaciones podrían desorientarlo totalmente. Por otra parte la construcción física de los móviles afecta el control que idealmente tendríamos sobre ellos 
(Han et al., 2005), por eso es necesario adicionar al modelo de control algunas variables que podrían no ser determinísticas.

Es necesario entonces desarrollar un sistema de posición que sea absoluto, y por eso se propone el uso de un sistema simple (periférico), de visión artificial, que permita observar todo el campo de trabajo de los robots móviles. En principio debe segmentar e individualizar cada Robot Móvil incluyendo una identificación del equipo al que pertenece además de ubicarlo correctamente en el espacio de trabajo. El sistema debe ser rápido, para reducir los problemas de predicción, y debe estimar el camino para cada móvil, teniendo en cuenta sus características físicas.

\section{Materiales Y MÉtodos}

\section{Conjuntos de prueba}

Dada la importancia de tener un conjunto de pruebas suficientemente reproducible, se decidió diseñarlo artificialmente para comprobar el rendimiento del algoritmo de segmentación y ubicación. Para ello se implementaron usando Flash MX ${ }^{\circledR} 20$ secuencias de video. Cada fotograma es de 768 x 1024 píxeles a una tasa de 24 fotogramas por segundo, igual al obtenido por la cámara digital. En todas las secuencias de video, intervienen los ocho móviles desplazándose a través de una trayectoria planeada usando interpolaciones polinómicas entre diferentes puntos del plano de trabajo.

\section{Selección del servomotor}

Aunque en esta fase del proyecto no fue necesario tener los prototipos totalmente construidos, era necesario determinar las características físicas del Robot Móvil, incluyendo el tipo y la marca de motores y las dimensiones. El servomotor que se escogió fue el mostrado en la Tabla 1. En este caso la decisión es muy importante, porque al determinar la velocidad máxima que el Robot Móvil puede desarrollar, se puede determinar la restricción espacial debida al tiempo en que el sistema se encuentra procesando la imagen. 
Tabla 1. Características del servomotor

\begin{tabular}{|l|l|}
\hline Marca & E-Sky \\
\hline Voltaje & DC $5 \mathrm{~V} \pm 1 \mathrm{~V}$ \\
\hline PWM & Ciclo $20 \pm 1 \mathrm{~ms}$, Pulso Positivo $1 \sim 2 \mathrm{~ms}$ \\
\hline Velocidad & Velocidad: $0.1 \mathrm{~S} / 60^{\circ}$ \\
\hline Torque & $1.0 \mathrm{Kg} / \mathrm{cm}(\mathrm{VCC}=5 \mathrm{~V})$ \\
\hline Peso & $7.5 \mathrm{~g}$ \\
\hline Dimensiones & $22.8 \times 11.5 \times 20.8 \mathrm{~mm}$ \\
\hline
\end{tabular}

\section{Robot Móvil: Características}

\section{Maniobrabilidad}

La maniobrabilidad es la facilidad con la que un vehículo es dirigido. En el caso de la Robótica Móvil está ligada a la arquitectura implementada en el diseño del prototipo, y a la consideración de algunas variables físicas como el rozamiento de las ruedas. Para los robots en configuración diferencial con ruedas libres omnidireccionales las ruedas deben tener diseños especiales que eviten que el eje de rotación se desplace. Por ejemplo se sugiere el uso de ruedas Suecas o ruedas libres esféricas (Siegwart \& Nourbakhsh, 2004).

\section{Controlabilidad}

El concepto de controlabilidad está asociado a la habilidad de usar un sistema en todo su espacio de funcionamiento, usando un nivel de acción acotado. En otras palabras es la posibilidad de llevar el Robot Móvil de un estado inicial a otro estado final con una acción de control admisible. La controlabilidad no implica que el estado final sea estable, solo que se puede alcanzar a partir de cualquier estado inicial (Ollero, 2001).

\section{Estabilidad}

La estabilidad en un Robot Móvil está asociada a la cantidad de fuerza necesaria para que pierda el equilibrio, bien sea estando en reposo o en movimiento. Aunque se puede pensar en el diseño 
de estructuras tipo monociclo, el sistema de control sería innecesariamente complicada para la mayoría de aplicaciones. Se tiene entonces que el mínimo de llantas necesarias para mantener con facilidad la estabilidad en reposo y en movimiento son 3 (Ollero, 2001).

\section{Segmentación}

Tomando en cuenta que el diseño de la cara superior de los Robots Móviles debe ajustarse a las reglas, se decidió seleccionar uno de los más comunes, teniendo en cuenta el tipo de segmentación que se implementó como se ve en la Figura 1.

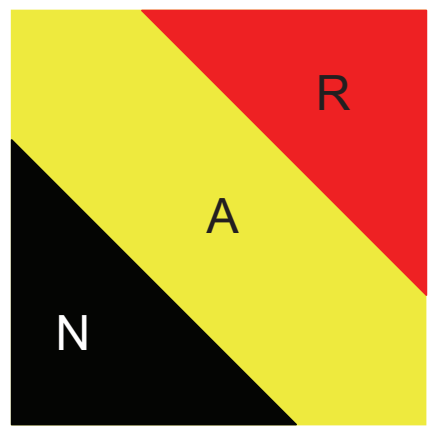

FIgURA 1. VISTA SUPERIOR DE LOS MÓVILES

El móvil contiene una sección superior a $3,5 \mathrm{~cm} \times 3,5 \mathrm{~cm}$ de color amarillo. El color rojo es un identificador de la posición de juego de cada móvil. En nuestro caso el color rojo es información usada para identificar individualmente cada robot. El color negro se usa para ser eliminado en la etapa de segmentación.

El algoritmo de segmentación usado, se encarga de crear una capa por cada móvil en el plano, es decir en cada capa creada, solo se observa el identificador individual de el robot Móvil correspondiente. Después de descomponer la matriz RGB y realizar una umbralización binaria sobre el fotograma podemos construir una tabla (Tabla 2) de verdad donde las salidas son las capas que se crean. 
En el fotograma resultante se presentan varios objetos a considerar. Para seleccionar el objeto correcto se escogió como característica el área a identificar que en este trabajo como en (Botero \& Delgado, 2009) es el triangulo que facilita particularmente el proceso de orientación del Robot Móvil.

TABLA 2. TABLA de Verdad SEgmentación.

\begin{tabular}{|c|c|c|l|c|c|c|c|c|c|c|c|}
\hline $\mathbf{R}$ & $\mathbf{G}$ & $\mathbf{B}$ & Color & $\boldsymbol{M}_{\boldsymbol{1}}$ & $\boldsymbol{M}_{\boldsymbol{2}}$ & $\boldsymbol{M}_{\boldsymbol{3}}$ & $\boldsymbol{M}_{\boldsymbol{4}}$ & $\boldsymbol{M}_{\boldsymbol{5}}$ & $\boldsymbol{M}_{\boldsymbol{6}}$ & $\boldsymbol{M}_{\mathbf{7}}$ & $\boldsymbol{M}_{\boldsymbol{8}}$ \\
\hline $\mathbf{0}$ & $\mathbf{0}$ & $\mathbf{0}$ & Negro & 0 & 0 & 0 & 0 & 0 & 0 & 0 & 0 \\
\hline $\mathbf{0}$ & $\mathbf{0}$ & $\mathbf{1}$ & Azul & 0 & 0 & 0 & 0 & 1 & 1 & 1 & 1 \\
\hline $\mathbf{0}$ & $\mathbf{1}$ & $\mathbf{0}$ & Verde & 1 & 0 & 0 & 0 & 1 & 0 & 0 & 0 \\
\hline $\mathbf{0}$ & $\mathbf{1}$ & $\mathbf{1}$ & Cian & 0 & 1 & 0 & 0 & 0 & 1 & 0 & 0 \\
\hline $\mathbf{1}$ & $\mathbf{0}$ & $\mathbf{0}$ & Rojo & 0 & 0 & 1 & 0 & 0 & 0 & 1 & 0 \\
\hline $\mathbf{1}$ & $\mathbf{0}$ & $\mathbf{1}$ & Magenta & 0 & 0 & 0 & 1 & 0 & 0 & 0 & 1 \\
\hline $\mathbf{1}$ & $\mathbf{1}$ & $\mathbf{0}$ & Amarillo & 1 & 1 & 1 & 1 & 0 & 0 & 0 & 0 \\
\hline $\mathbf{1}$ & $\mathbf{1}$ & $\mathbf{1}$ & Blanco & 0 & 0 & 0 & 0 & 0 & 0 & 0 & 0 \\
\hline
\end{tabular}

Se simplifican las funciones lógicas correspondientes a cada capa, y se obtienen las funciones de salida como se muestra en (1):

$$
\begin{aligned}
& M_{1}=R^{\prime} G B^{\prime}+R G B^{\prime} \\
& M_{2}=R^{\prime} G B+R G B^{\prime} \\
& M_{3}=R G^{\prime} B^{\prime}+R G B^{\prime} \\
& M_{4}=R G^{\prime} B+R G B^{\prime} \\
& M_{5}=R^{\prime} G B^{\prime}+R^{\prime} G^{\prime} B \\
& M_{6}=R^{\prime} G B+R^{\prime} G^{\prime} B \\
& M_{7}=R G^{\prime} B^{\prime}+R^{\prime} G^{\prime} B \\
& M_{8}=R G^{\prime} B+R^{\prime} G^{\prime} B
\end{aligned}
$$

Las operaciones lógicas dan como resultado una matriz binaria de tamaño $\mathrm{m} \times \mathrm{n},\left(M_{1}, M_{2}, M_{3}, M_{4}, M_{5}, M_{6}, M_{7}, M_{8}\right)$ en cada matriz como se había mencionado, se identifican varios objetos (crecimiento de regiones), y se efectúa una selección teniendo en cuenta su área. En la Tabla 3 se muestra la comparación de los 
posibles objetos identificados y sus áreas. Es necesario destacar que es posible implementar otro tipo de umbralizaciones que permitan identificar un número mayor de Robots Móviles.

TABLA 3. PROPORCIÓN DE OBJETOS IDENTIFICADOS

\begin{tabular}{|l|c|c|}
\hline \multicolumn{1}{|c|}{ Tipo de objeto } & Área en función del lado & Área en porcentaje \\
\hline Triangulo & $\frac{30.25 \times a^{2}}{128}$ & $23.63 \%$ \\
\hline Franja central & $\frac{33.75 \times a^{2}}{64}$ & $52.73 \%$ \\
\hline $\begin{array}{l}\text { Triangulo y fran- } \\
\text { ja central }\end{array}$ & $\frac{97.75 \times a^{2}}{128}$ & $76.37 \%$ \\
\hline
\end{tabular}

Usando las proporciones descritas en la Tabla 3 el algoritmo selecciona el área que corresponde al móvil que se espera identificar. Las proporciones son amplias para dar al algoritmo robustez a los problemas de adquisición del sistema.

\section{Ubicación y orientación}

Después de tener identificado el triángulo asociado a cada Robot Móvil, la siguiente tarea consiste en obtener su posición y orientación basándose en el sistema de referencia que provee el campo de trabajo de la cámara como se muestra en la Figura 2.

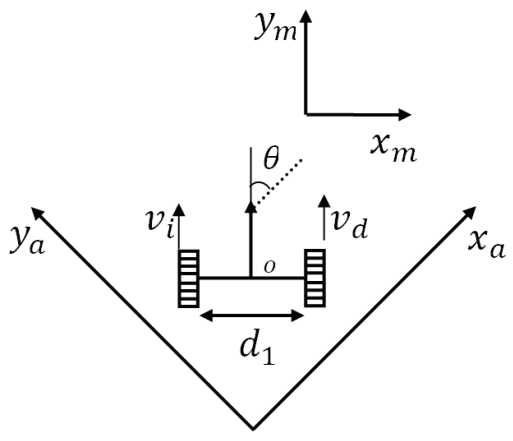

Figura 2. Posicionamiento del Robot Móvil 
Al obtener el centro del triángulo que segmenta el algoritmo descrito, no se obtiene el centro de masa del Robot Móvil, por eso es necesario realizar una transformación. Partiendo del modelo de vista superior mostrado en la Figura 3 , donde $c_{1}=\left(x_{1}, y_{1}\right)$ corresponde al centro calculado en la fase de segmentación y $c_{2}=\left(x_{2}, y_{2}\right)$ corresponde al centro real del móvil necesario para desarrollar el modelo cinemático. Por tanto se tiene que determinar una matriz de transformación que desplace y rote el punto $c_{2}$ al punto $c_{1}$. Como los móviles se desplazan sobre un plano (2D), las matrices de traslación y rotación están dadas por (2).

$$
\begin{aligned}
& T=\left[\begin{array}{lll}
1 & 0 & t_{x} \\
0 & 1 & t_{y} \\
0 & 0 & 1
\end{array}\right] \\
& R=\left[\begin{array}{ccc}
\cos \theta & -\sin \theta & 0 \\
\sin \theta & \cos \theta & 0 \\
0 & 0 & 1
\end{array}\right]
\end{aligned}
$$

Donde $\left(t_{x}, t_{y}\right)$ indican la magnitud de la traslación y $\theta$ el ángulo de rotación.

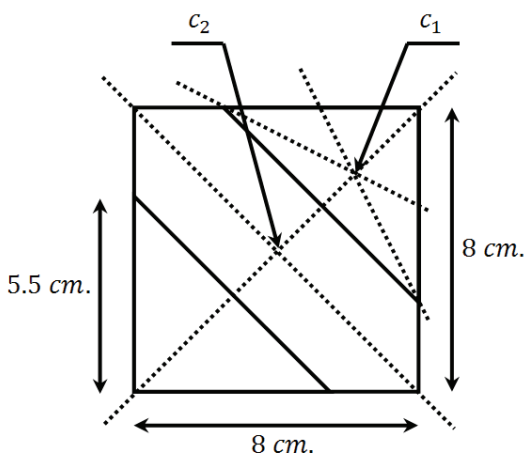

Figura 3. MedidAs de LA VISTA SUPERIOR

En función de $c_{1}=\left(x_{1}, y_{1}\right)$ y tomando las medidas de forma simbólica se pueden obtener las matrices de transformación para trasladar el vector director encontrado en la fase de segmentación, al vector director real del móvil, las matrices se muestran en (3). 


$$
\begin{aligned}
& T_{1}=\left[\begin{array}{ccc}
1 & 0 & -x_{1} \\
0 & 1 & -y_{1} \\
0 & 0 & 1
\end{array}\right] R_{1}=\left[\begin{array}{ccc}
\cos \frac{\pi}{4} & -\sin \frac{\pi}{4} & 0 \\
\sin \frac{\pi}{4} & \cos \frac{\pi}{4} & 0 \\
0 & 0 & 1
\end{array}\right] \\
& T_{2}=\left[\begin{array}{ccc}
1 & 0 & x_{1}-\sqrt{\left(\frac{a}{2}-\frac{b}{3}\right)^{2}+\left(\frac{a}{2}-\frac{b}{3}\right)^{2}} \cos \theta \\
0 & 1 & y_{1}-\sqrt{\left(\frac{a}{2}-\frac{b}{3}\right)^{2}+\left(\frac{a}{2}-\frac{b}{3}\right)^{2}} \sin \theta \\
0 & 0 & 1
\end{array}\right]
\end{aligned}
$$

Donde $\alpha$ representa la longitud del lado del cuadrado mostrado en la Figura $3(8 \mathrm{~cm})$, y $b$ representa la longitud de los catetos del triángulo identificado. En (3), $T_{1}$ es la matriz que expresa traslación al origen, $R_{1}$ la rotación inicial y $T_{2}$ la traslación al centro de masa del Robot Móvil. Después de encontrar las matrices se obtiene la matriz de transformación general como se expresa en (4).

$$
T=T_{2} R_{1} T_{1}
$$

\section{Evasión}

Cuando se conoce la dirección y la posición de un Robot Móvil se puede determinar la ecuación de la recta que seguirá. Como en este caso se trata de un sistema físico de procesamiento con restricciones, no se puede determinar una colisión simplemente con la intersección de dos rectas. Se debe entonces considerar el tiempo de procesamiento y la velocidad que el Robot Móvil puede desarrollar como se expresa en (5).

$$
\begin{gathered}
v=0.2105 \frac{\mathrm{m}}{\mathrm{s}} \\
D_{1}=v t=0.3 s \quad 0.2105 \frac{\mathrm{m}}{\mathrm{s}}=0.0631 \mathrm{~m}=0.717 \mathrm{a}
\end{gathered}
$$


Donde $v$ es la velocidad máxima que el Robot Móvil puede desarrollar, y $t$ el tiempo máximo de procesamiento medido en Matlab® para segmentar y ubicar los móviles usando una cámara digital de 640x480 píxeles en un Procesador Pentium D a $3 \mathrm{GHz}$ con $1.00 G B$ de RAM. Adicionalmente es necesario determinar la restricción debida a las dimensiones del Robot Móvil, es decir respetar el circulo que lo inscribe. Como se muestra en (6), es necesario desarrollar los algoritmos de forma simbólica para poder escalarlos a futuras aplicaciones, y para poder calibrar fácilmente el sistema ante cambios en sus partes.

$$
D_{2}=\frac{\sqrt{2}}{2} a
$$

Con base en lo anterior se desarrollo el algoritmo de evasión determinando la posición que cada móvil tendrá en el tiempo en que no se realiza procesamiento, se muestra en la Figura 4.

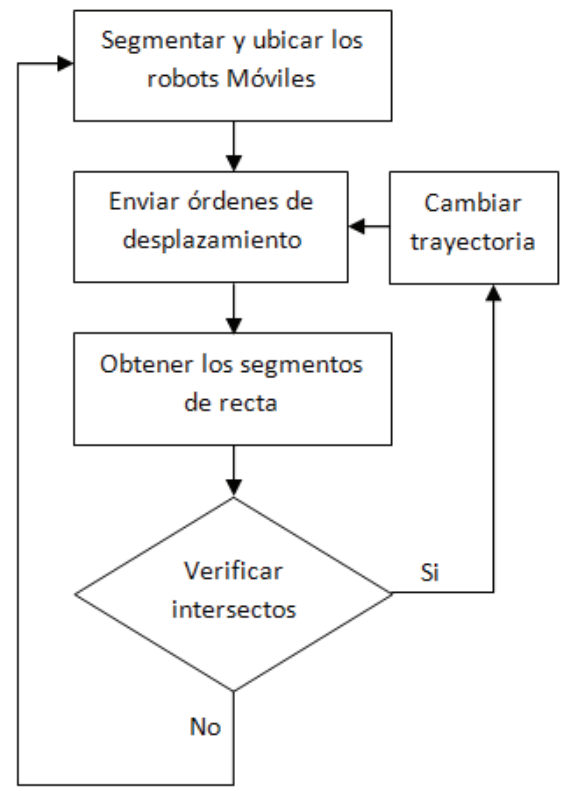

Figura 4. Algoritmo de eVAsión 


\section{Resultados y discusión}

En la Figura 5, se presenta una escena estática para evaluar la efectividad del cálculo del área. Se observan dos equipos de cuatro móviles cada uno, el cuadro azul y amarillo en la parte inferior es usado para calibrar el área total de un móvil.

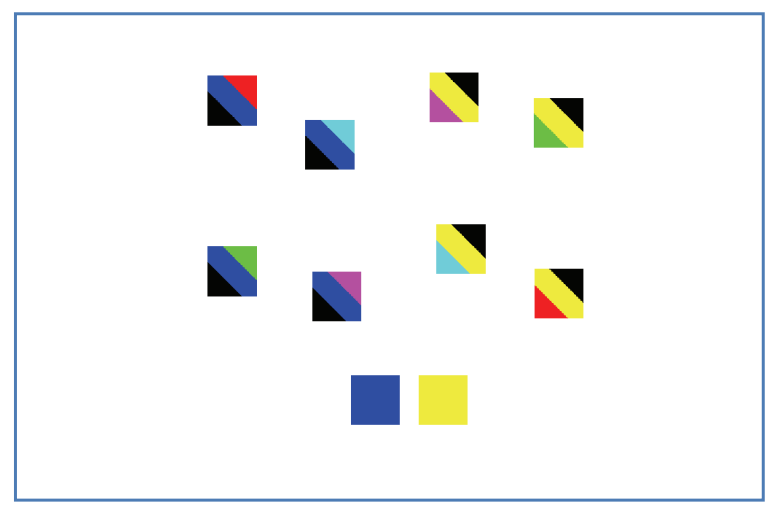

FigURA 5. EqUiPOS DE PRUEBA.

En la Figura 6 se muestra el fotograma resultante después de umbralizar y aplicar la operación lógica correspondiente a $M_{1}$ mostrada en (1). En la Figura 7 se muestra el resultado de la capa $M_{5}$.

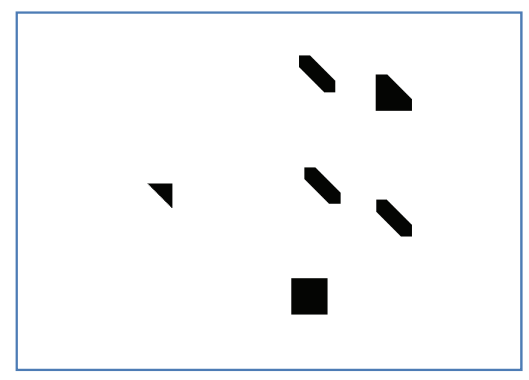

Figura 6. FunCIÓN $M_{1}$ OBTENIDA A PARTIR DE (1)

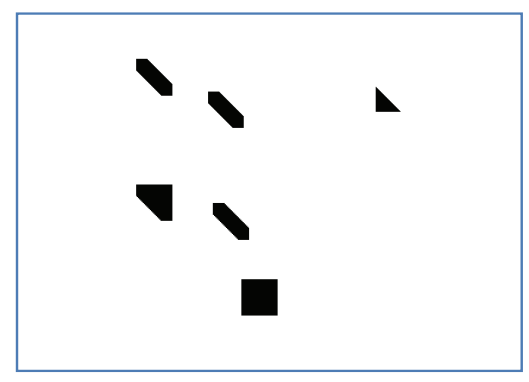

FIgURA 7. FUNCIÓN $M_{5}$ OBTENIDA A PARTIR DE (1) 
Para probar la robustez del algoritmo ante condiciones de ruido añadimos Ruido Sal y Pimienta al $20 \%$, se muestra en la Figura 8. En la Figura 6 se muestra el fotograma resultante después de umbralizar y aplicar la operación lógica correspondiente a $M_{1}$.

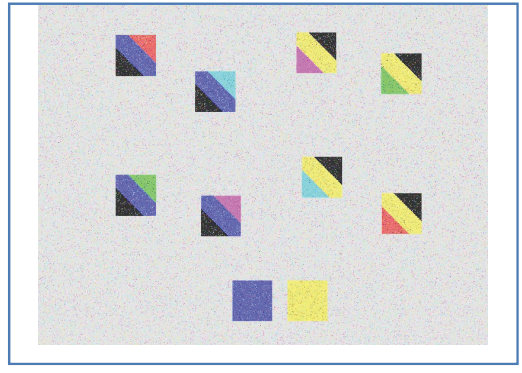

Figura 8. Fotograma con RUIDO SAL Y PIMIENTA

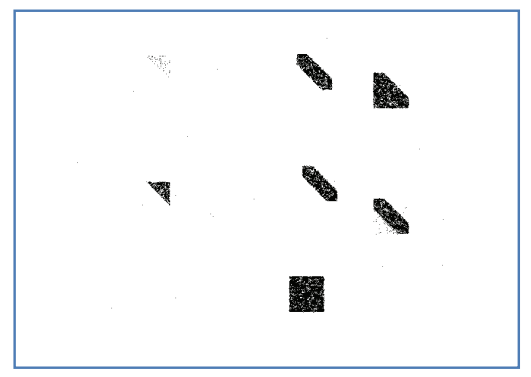

Figura 9. MóviL 5 CON RUIDO SAL Y PIMIENTA

En la Tabla 4 se muestra la comparación entre las áreas calculadas geométricamente (mostradas en la Tabla 3 con las áreas encontradas por el algoritmo de segmentación en el fotograma de prueba y el fotograma con ruido Sal y Pimienta. Es claro que el error es bajo y la diferencia de áreas entre los objetos identificados permite seleccionar el móvil con facilidad.

TABLA 4. Comparación de LAS ÁREAS CALCULAdAS

\begin{tabular}{|l|c|c|c|c|c|}
\hline Tipo de objeto & AFP & AFR & APC & EFP & EFR \\
\hline Área total & 8372 & 7862 & $100 \%$ & - & - \\
\hline Triángulo & 2016 & 2350 & $23.63 \%$ & $1.88 \%$ & $6.26 \%$ \\
\hline Franja central & 4403 & 4190 & $52.73 \%$ & $0.25 \%$ & $0.56 \%$ \\
\hline Triángulo y franja central & 6448 & 6232 & $76.37 \%$ & $0.85 \%$ & $2.90 \%$ \\
\hline
\end{tabular}

Donde AFP es el área en pixeles del fotograma de prueba, AFR es el área en pixeles del fotograma de con ruido, APC el área en porcentaje calculado geométricamente, EFP es el error del fotograma de prueba con respecto al APC y EFR es el error del fotograma ruido con respecto al APC. 


\section{Conclusiones}

1. Mediante operaciones sobre imágenes binarias se logró la segmentación individual y la ubicación absoluta en el plano de un conjunto de Robots Móviles. El sistema de localización y orientación implementado es efectivo en entornos de trabajo controlados, la iluminación no difusa o el uso de equipos de adquisición de mala calidad haría necesaria la implementación de técnicas de filtrado.

2. El algoritmo de segmentación implementado permite ejecutar acciones sobre los Robos Móviles en tiempo real. Siendo así, la planeación de las trayectorias se pudo realizar dinámicamente, aunque las limitaciones en la velocidad de procesamiento pueden añadir tiempos muertos en los que el sistema no tendría información sobre el espacio de trabajo.

3. La forma escogida para la segmentación permitió conocer directamente la orientación y posición del Robot Móvil a través de operaciones matriciales simples. Sin embargo se podrían presentar confusiones si los robots móviles se encuentran demasiado cerca.

4. El sistema de ubicación absoluto desarrollado posiciona los Robots, aun cuando su estado sea forzado externamente, no obstante hace necesario procesar toda la imagen en cada secuencia de video.

\section{Referencias}

Arias, L. D., \& León, P. A. (2007). Identificación de Posición y Dirección de Objetos en en R2 Usando Color. XIII Simposio de Tratamiento de Señales, Imágenes y Visión Artificial, (pág. 5). Bucaramanga.

Botero Valencia, J. S., \& Delgado Trejos, E. (2009). Segmentación para la identificación y planeación de trayectorias de Robots en 2D sobre secuencias de video. XIV Simposio de Tratamiento de Señales, Imágenes y Visión Artificial. Pereira: Universidad Tecnológica de Pereira. 
Chen, S., Chng, E. S., \& Alkadhimi, K. (1996). Regularized orthogonal least squares algorithm for constructing radial basis function networks. International Journal in Control , 829-837.

Duda, R. O., Hart, P. E., \& Stork, D. G. (2000). Pattern Classification. New York: Wiley.

FIRA. (Julio de 2009). MiroSot rules. Obtenido de FIRA.

Gonzalez, R. C., \& Woods, R. E. (2008). Digital Image Processing (Tercera Edición ed.). New Jersey, United States: Prentice-Hall.

Han, M., Kopacek, P., Putz, B., Sshierer, E., \& M., W. (2005). Mobile Mini Robots for MAS. En M. A. Armada, P. de González Santos, \& S. Berlín (Ed.), Climbing and Walking Robots. Berlín: Springer.

Han, M.-W., \& Kopacek, P. (2007). Mini Robots for Soccer . En R. Moreno Díaz, F. Pichler, A. (. Quesada Arencibia, \& S. Berlin (Ed.), Computer Aided Systems Theory (págs. 1009-1016). Berlín: Springer.

Haykin, S. (1999). Neural Networks: A Comprehensive Foundation . New York: Prentice-Hall.

Hernandez L., J., \& Salazar G., S. (2006). Implementación de una Maquina de Vectores de Soporte empleando FPGA. Scientia et Technica Año XII, No $31,47-52$.

Hush, D. R., \& Horne, B. (1998). Efficient algorithms for function approximation with piecewise linear sigmoidal networks. IEEE Transaction on Neural Networks , 1129-1141.

Kondo, N., Hatanaka, T., \& Uosaki, K. (2006). Pattern Classification via Multi-objective Evolutionary RBF Networks. SICE-ICASE International Joint Conference, 137-142.

(2007). En G. Minati, E. Pessa, \& S. US (Ed.), Collective Beings (págs. 321 352). US: Springer.

Ollero Baturone, A. (2001). Robótica manipuladores y robots móviles (Primera edición ed.). Barcelona, España: Marcombo.

Ortíz A., L. F., Muñoz C., N. D., \& Londoño Ospina, N. (2008). Sistema de localización de bajo costo y alta prestación para una plataforma robótica móvil. Scientia et Technica , XIV (39), 6.

Schilling, R. J., Carroll, J. J., \& Al-Ajlouni, A. F. (2001). Approximation of nonlinear systems with radial basis function neural network. IEEE Transactions on Neural Networks, 1-15. 
Schwenker, F., Kestler, H. A., \& Palm, G. (2000). Radial-Basis-Function Networks: Learning and Applications. Fourth International Conference on knowledge-Based Intelligent Engineerings Systems \& Allied Technologies, 33-43.

Shoham, Y., \& Leyton-Brown, K. (2009). Multiagent Systems: Algorithmic, Game-Theoretic, and Logical Foundations (Primera edición ed.). New York, United States: Cambridge University Press.

Siegwart, R., \& Nourbakhsh, I. R. (2004). Introduction to Autonomous Mobile Robots (First edition ed.). United States of America: MIT Press.

Tinós, R., \& Murta Júnior, L. O. (2008). Selection of Radial Basis Functions via Genetic Algorithms in Pattern. 10th Brazilian Symposium on Neural Networks, 171-176.

Witten, I. H., \& Frank, E. (2005). Data Mining: Practical Machine Learning Tools and Techniques. San Francisco: Morgan Kaufman.

Zhang, W., He, M., \& Mak, M. W. (2001). Application of MLP and RBF Networks to cloud detection. Proceedings of 2001 International Symposium on Intelligent Multimedia, Video and Speech Processing , 60-63. 